\title{
Role of Point-of-care Ultrasound in Management of Critically Ill COVID-19 Patients: A Case Series
}

Indranil Biswas ${ }^{1}$, Krishna P Gourav², Dheemta Toshkhani ${ }^{3}$, Ashish Agarwal ${ }^{4}$, Suresh K Angurana ${ }^{5}$, Jayashree Muralidharan ${ }^{6}$, Atit A Gawalkar ${ }^{7}$, Manoj K Rohit ${ }^{8}$, Vivek Jaswal ${ }^{9}$, Goverdhan D Puri ${ }^{10}$

\begin{abstract}
Introduction: Hereby, we describe a series of four critically ill COVID-19 patients where point-of-care ultrasound (POCUS) helped in guiding specific management.

Case description: The first case is a 62-year-old COVID-19 positive woman where severe aortic stenosis and severe left ventricular dysfunction were diagnosed by POCUS, which led to the institution of specific medical management leading to resolution of her symptoms and referral to the cardiac surgical department for further surgical management. The second case is a 51-year-old woman admitted with severe hypoxia secondary to COVID-19 infection. She was being considered for tocilizumab therapy. However, POCUS revealed the presence of pericardial effusion, which, on evaluation, was found to be due to tubercular. This led to withholding tocilizumab therapy. Anti-tubercular therapy, instead, was instituted. The third case is a 13-month-old child, who presented with a history of recurrent syncopes and was diagnosed as a case of congenital heart block on electrocardiography (ECG). However, due to his COVID-19 positive status, rescue temporary pacing could not be performed at the catheterization laboratory. Point-of-care ultrasound helped in the successful placement of a temporary pacemaker lead at the bedside, leading to the achievement of optimum heart rate till he got an epicardial pacemaker inserted at a later date. The fourth case is of a 45-year-old man, who had to undergo endotracheal intubation due to refractory COVID-19 related hypoxia. Upon connection to the mechanical ventilator, the peak airway pressure was found to be unusually high. On POCUS, lung sliding on the left side was missing, which led to the diagnosis of rightmainstem endobronchial intubation. Repositioning of the endotracheal tube led to a decrease in peak airway pressures and optimal delivery of mechanical ventilation to the patient.

Conclusion: Point-of-care ultrasound can help diagnose and manage significant underlying diseases, help take/modify decisions on specific therapies, and overcome resource limitations for performing specialized therapeutic procedures in COVID-19 patients.

Keywords: Aortic stenosis, Complete heart block, COVID-19, Echocardiography, Lung ultrasound, Point-of-care ultrasound, Tocilizumab. Journal of Perioperative Echocardiography (2019): 10.5005/jp-journals-10034-1104
\end{abstract}

\section{INTRODUCTION}

Point-of-care ultrasound (POCUS) is an indispensable part of critical care management. It can rapidly provide important and relevant information at the bedside, ${ }^{1}$ thus avoiding delays in diagnosis and initiation of appropriate management. ${ }^{2}$ Guidelines by several societies also have recommended the use of ultrasound at the bedside for diagnostic assessment, therapeutic decision-making as well as for procedural guidance. ${ }^{3-5}$

India is at present the second worst-hit country in the world by the ongoing COVID-19 pandemic with almost 8 million confirmed cases. ${ }^{6}$ This huge burden on an already resource-limited healthcare system leads to the severe restriction upon the critically ill COVID19 patients to access optimal diagnostic and therapeutic services. Point-of-care ultrasound has huge potential in this resourceconstrained scenario as a simple, low-cost, diagnostic tool with therapeutic applications, too.

We are hereby reporting four cases where POCUS helped in diagnosing specific disease/condition at the bedside, helped in specific therapeutic decision-making, and guided administration of specialized intervention in critically ill COVID-19 patients in a tertiary level COVID care hospital in India.

\section{Case Descriptions}

\section{Case 1}

A 79-year-old woman was admitted with a history of fever and shortness of breath for the last 4 days. On examination, she had
${ }^{1-3,10}$ Department of Anesthesia and Intensive Care, Postgraduate Institute of Medical Education and Research, Chandigarh, India

${ }^{4-6}$ Department of Pediatrics, Postgraduate Institute of Medical Education and Research, Chandigarh, India

${ }^{7,8}$ Department of Cardiology, Postgraduate Institute of Medical Education and Research, Chandigarh, India

${ }^{9}$ Department of Cardiothoracic and Vascular Surgery, Postgraduate Institute of Medical Education and Research, Chandigarh, India

Corresponding Author: Indranil Biswas, Department of Anesthesia and Intensive Care, Postgraduate Institute of Medical Education and Research, Chandigarh, India, Phone: +91 7710449858, e-mail: hreesheekombartta@gmail.com

How to cite this article: Biswas I, Gourav KP, Toshkhani D, et al. Role of Point-of-care Ultrasound in Management of Critically III COVID-19 Patients: A Case Series. J Perioper Echocardiogr 2019;7(2):40-43.

Source of support: Nil

Conflict of interest: None

$84 \%$ saturation by pulse oximetry in room air. Real-time polymerase chain reaction (RT-PCR) of her nasopharyngeal swab came back as positive for severe acute respiratory syndrome coronavirus type 2 (SARS-CoV-2). She was shifted to the intensive care unit (ICU) of the isolation facility of our institute. On detailed history taking, she revealed the presence of New York Heart Association (NYHA) grade 2 dyspnea on exertion for the last 2 years. On examination, she was 
normotensive with mild tachycardia of $105 \mathrm{bpm}$. Her pulse was regular and slow rising. On bedside point-of-care echocardiography, her aortic valve was found to be severely calcific and stenotic (Fig. 1). The mean gradient across the aortic valve was found to be $43 \mathrm{~mm} \mathrm{Hg}$ along with severe left ventricular systolic dysfunction (left ventricular ejection fraction 30\%) and dilated ascending aorta $(4.6 \mathrm{~cm})$ (Fig. 1 and Video 1).

Besides low-molecular-weight heparin, she was started on metoprolol, spironolactone, and furosemide. Gradually, her heart rate decreased and settled at $68 \mathrm{bpm}$ on the 4th day of admission. Her $\mathrm{O}_{2}$ requirement decreased gradually from $6 \mathrm{~L}$ /minute flow by nasal prongs at the time of admission to room air on the 6th day of ICU stay. She was discharged to home on the 21st day of admission after RT-PCR from her nasopharyngeal swab turned negative for SARS-CoV-2. She was planned for an aortic valve replacement with/ without ascending aortic intervention electively.

\section{Case 2}

A 64-year-old diabetic and hypertensive lady presented with a history of fever and shortness of breath for the last 4 days and loose stools for the last 1 day with her nasopharyngeal swab tested positive for SARS-CoV-2 by RT-PCR. Although hemodynamically stable, she was tachypneic with a respiratory rate of 45-50/ minute and maintaining saturation of $91 \%$ with 0.9 inspired fraction of $\mathrm{O}_{2}$ by high-flow nasal cannula with a poor $\mathrm{P}_{\mathrm{a}} \mathrm{O}_{2} / \mathrm{F}_{\mathrm{i}} \mathrm{O}_{2}$ ratio of 55-60. The blood markers of disease severity were also high for her with a neutrophil-lymphocyte ratio (N-L ratio) of 23 , C-reactive protein (CRP) of $135 \mathrm{mg} / \mathrm{L}$, and D-dimer of 1,226 ng/ $\mathrm{mL}$. Her interleukin-6 levels were also high $-257 \mathrm{ng} / \mathrm{mL}$. She had a normal procalcitonin level and normal renal as well as liver functions. Based on her clinical condition and laboratory parameters, she was being considered for a trial of tocilizumab therapy. On a screening point-of-care echocardiography, she was found to have a normal biventricular function with moderate pericardial effusion (Fig. 2). The pericardial fluid was aspirated for diagnostic studies. On finding high adenosine deaminase level in the pericardial fluid, it was sent for detection of Mycobacterium tuberculosis, which came back as positive. Due to the presence of active tuberculosis infection, the tocilizumab therapy was withheld and antitubercular therapy was started. She later had an ischemic stroke leading to left-sided hemiplegia on the 12th day of admission. Her oxygen requirement, however, came down gradually and she was on room air by the 14th day of admission. She was discharged home on the 21st day of admission with residual left-sided hemiparesis.

\section{Case 3}

A 13-month-old child presented to our institute with respiratory failure, hypotensive shock, and encephalopathy. She had a history of recurrent syncopes for the last 3 months. Due to the imposition of nationwide lockdown and restriction of access to healthcare for non-COVID-19 illnesses, it could not be evaluated properly. She was admitted to our institute on mechanical ventilation and vasoactive support. Her heart rate was 55 /minute at presentation with complete heart block on electrocardiogram (ECG) (Fig. 3). She was started on isoprenaline infusion and was planned for permanent pacemaker insertion. However, her nasopharyngeal swab tested positive for SARS-CoV-2 by RT-PCR. She was shifted to a dedicated pediatric COVID-19 critical care unit. In view of worsening heart rate (40/minute), shock, renal function, and high-anion gap metabolic acidosis, peritoneal dialysis was initiated and temporary pacemaker insertion was planned. As the dedicated COVID-19 facility at our institute did not have a dedicated catheterization laboratory at that time and the regular catheterization laboratory of the institute could not be utilized for procedures on COVID-19 patients, a temporary transvenous pacemaker wire was inserted via the right internal jugular vein access at the bedside under point-of-care echocardiographic guidance (Fig. 4). Point-of-care transthoracic echocardiography helped in guiding the placement of the pacemaker wire into proper position inside the right ventricle (RV). It also led to the detection of a patent ductus arteriosus (PDA) in the patient. Following the initiation of pacing, there was an improvement in heart rate, hemodynamics, and renal function and there was a resolution of metabolic acidosis. The child was gradually weaned off from vasoactive support, peritoneal dialysis, and mechanical ventilation. She was extubated on the 16th day of admission and was discharged from the critical care unit. She was discharged from the dedicated COVID-19 facility after she tested negative for SARS-CoV-2. Later on, she underwent surgical permanent pacemaker insertion and PDA ligation on the 22nd day
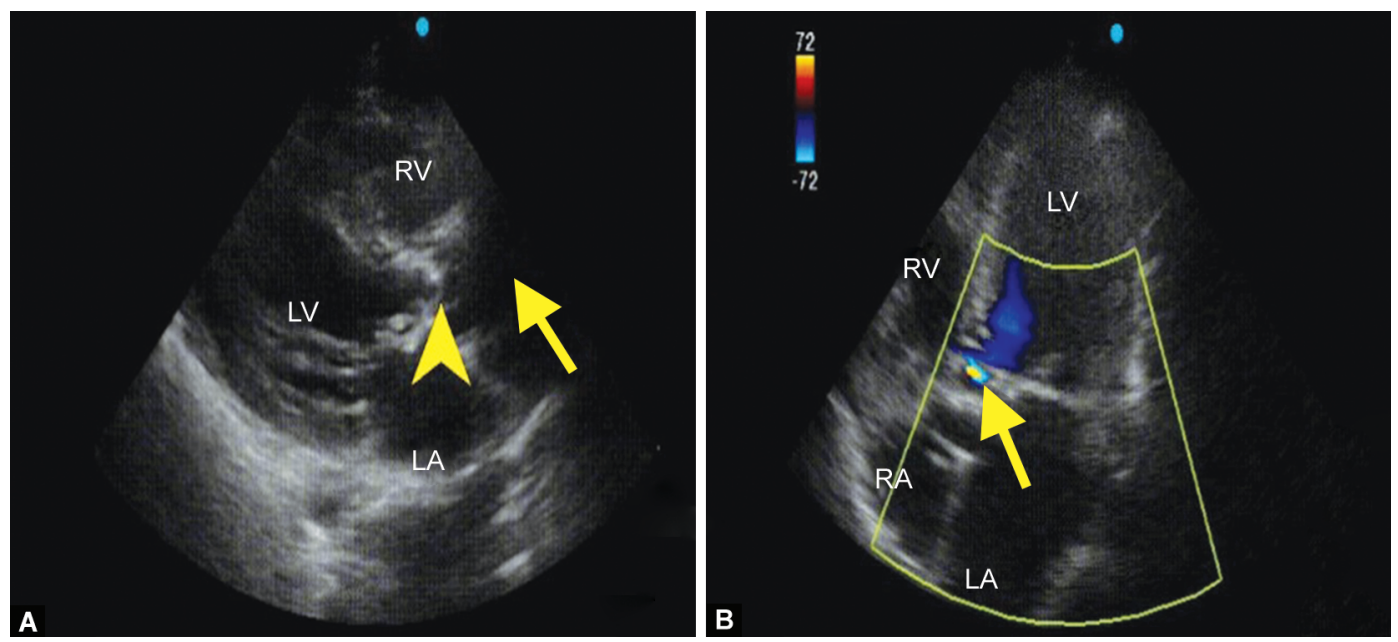

Figs 1A and B: (A) Parasternal long-axis view showing severely calcified aortic valve (yellow arrowhead) and dilated ascending aorta (yellow arrow); (B) Apical four-chamber view color Doppler showing flow acceleration proximal to the aortic valve (yellow arrow). LA, left atrium; RA, right atrium; LV, left ventricle; RV, right ventricle 


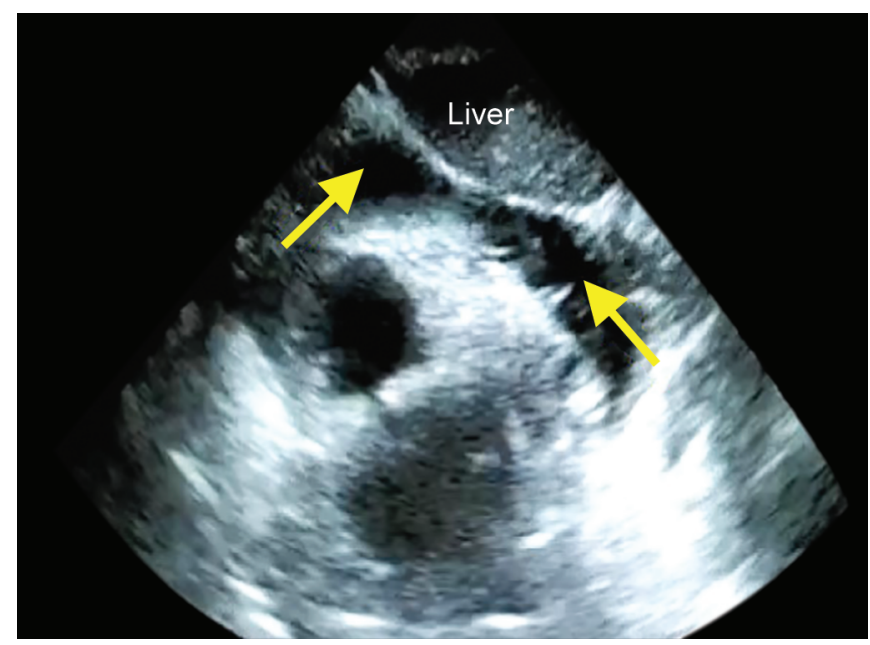

Fig. 2: Subcostal view showing the presence of pericardial effusion (yellow arrows)

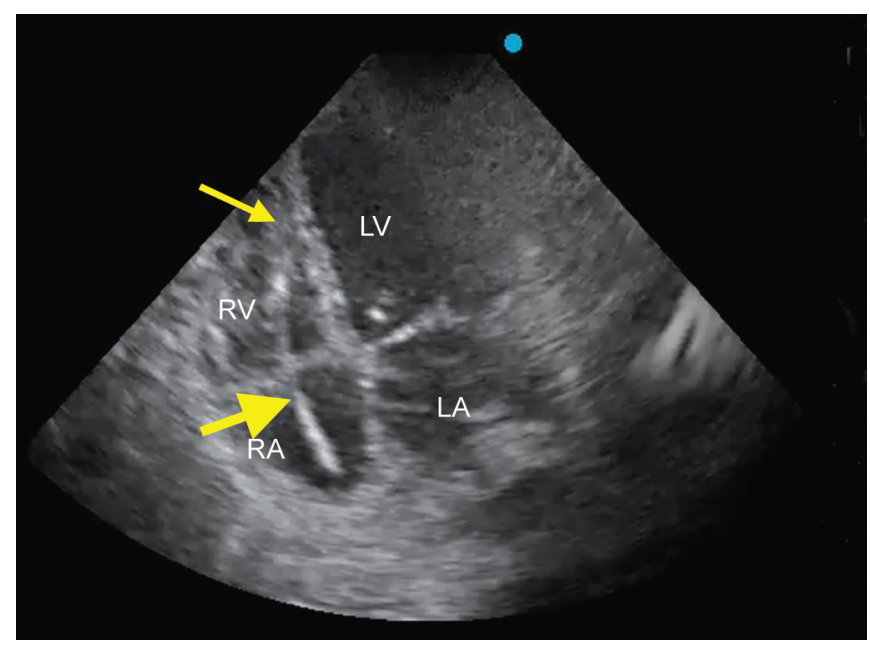

Fig. 4: Right ventricle focused apical four-chamber view showing the position of temporary pacemaker wire (yellow thick arrow) within cardiac chambers with its tip (yellow thin arrow) positioned within RV. LA, left atrium; RA, right atrium; LV, left ventricle; RV, right ventricle

of admission and was subsequently discharged uneventfully to home on the 30th day of admission.

\section{Case 4}

A 45-year-old, hypertensive, diabetic, non-obese man presented with shortness of breath and fever for 4 days. His chest X-ray was suggestive of COVID ARDS and his nasopharyngeal swab tested positive for SARS-CoV-2 by RT-PCR. Due to worsening P/F ratio (55) and persistent tachypnea (55/minute) on non-invasive ventilation, endotracheal intubation was performed and he was put on invasive mechanical ventilation. The endotracheal intubation was performed using a C-MAC videolaryngoscope and the endotracheal tube was fixed at $22 \mathrm{~cm}$ at the level of the incisors. However, on the initiation of mechanical ventilation, the peak airway pressure reached $52 \mathrm{~cm} \mathrm{H}_{2} \mathrm{O}$ with a tidal volume of $6 \mathrm{~mL} / \mathrm{kg}$, respiratory rate of 32 , and positive end-expiratory pressure (PEEP) of $10 \mathrm{~cm} \mathrm{H}_{2} \mathrm{O}$. As auscultation is not possible while wearing full personal protective equipment (PPE) and chest X-ray was not immediately available,

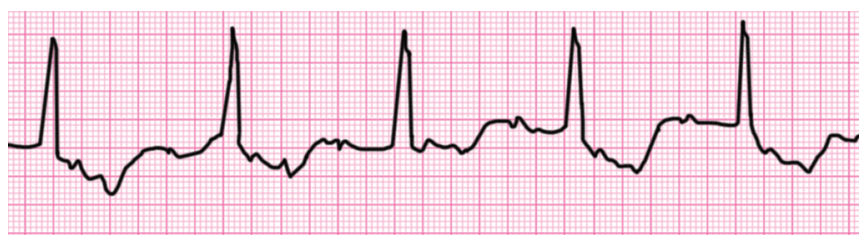

Fig. 3: Electrocardiogram showing complete heart block

point-of-care lung ultrasound was performed. It showed grossly reduced lung sliding on the left side, however, no "lung point" or "stratosphere sign" was visible (Video 2A). There was normal lung sliding on the right side (Video 2B). Based on the finding, right mainstem endobronchial intubation was suspected and the endotracheal tube was re-fixed at $20 \mathrm{~cm}$ at the level of the incisors. The peak airway pressure reduced to $38 \mathrm{~cm} \mathrm{H}_{2} \mathrm{O}$ and a repeat lung ultrasound showed normal lung sliding on both sides of the chest. A subsequent chest $X$-ray showed the tip of the endotracheal tube located $1 \mathrm{~cm}$ proximal to the carina.

\section{Discussion}

A cardiac examination is often the most common indication for POCUS. ${ }^{7}$ In a study done over 6 months, bedside echocardiography has changed management in up to $50 \%$ of patients, including identifying the previously unknown underlying cardiac disease. ${ }^{8}$ In our first case, apart from helping in the assessment of volume status and guidance for fluid management, bedside echocardiography helped in identifying previously unknown diagnosis of severe aortic valve stenosis and severe left ventricular systolic dysfunction. This led to the institution of specific medical management in the form of heart rate control by beta-blocker therapy and pulmonary decongestion by diuretics. This led to rapid clinical improvement in the patient and expedited her recovery.

The presence of a dedicated bedside ultrasound machine along with the presence of a dedicated, compatible echocardiography probe and regular posting of a trained cardiac anesthesiology fellow in the ICU of the dedicated COVID-19 hospital of our institute allows the performance of routine comprehensive echocardiography of all the patients admitted in the ICU. An 18-month survey done in 704 patients admitted in general ICU has previously revealed that routine comprehensive echocardiography diagnosed a clinically significant cardiac problem that was previously unknown in onethird of patients. ${ }^{9}$ In our second patient, routine comprehensive echocardiography revealed previously unknown and unsuspected pericardial effusion in the patient. The effusion, on evaluation, was found to be of tubercular origin. This posed as a contra-indication to tocilizumab therapy ${ }^{10}$ that was being considered in this patient, based upon her clinical condition and high levels of disease severity markers. Thus, POCUS led to significant modification of therapeutic strategy in this COVID-19 patient.

Procedural guidance is one of the most recommended indications for use of POCUS in ICU. ${ }^{5,11}$ Although procedures like vascular access, pericardiocentesis, and thoracentesis are commonly performed in the ICUs under ultrasound guidance, highly specialized procedures like transvenous pacemaker insertion have also been reported being done under echocardiographic guidance. ${ }^{12}$ In our third case, POCUS played an immensely important role in guiding the insertion of transvenous pacemaker wire on an urgent basis. Due to severe resource limitation, the catheterization laboratory of our institute was, at that time, not prepared for usage 
for COVID-19 patients. This underscores the importance of POCUS in that it can overcome the shortcomings of resource limitation, especially at this time of an enraging pandemic.

Lung ultrasound is usually the second most commonly used POCUS after echocardiography. ${ }^{7}$ It helps in diagnosing pneumothorax, pleural effusion, lung consolidation, atelectasis, and various conditions affecting pleura and lung parenchyma. ${ }^{13-16}$ Its use is associated with reduced requirements for chest radiograph and computed tomography. ${ }^{7}$ In our fourth case, the use of bedside lung ultrasound helped in quick troubleshooting of the scenario by diagnosing endobronchial intubation, thus replacing the need for auscultation which is impossible while donned in full PPE and also overcoming the immediate nonavailability of the portable chest radiograph in the COVID-19 hospital.

\section{Conclusion}

Amid COVID-19 pandemic which is causing an enormous burden on the already resource-constrained healthcare system like that of India, POCUS is a simple, cost-effective technology which can help diagnose and manage significant underlying diseases, help take/modify decision on specific therapies, and overcome resource limitations for performing specialized therapeutic procedures in COVID-19 patients.

Video 1: Apical five-chamber view showing severely calcified aortic valve (yellow arrow) and LV systolic dysfunction. LA: Left atrium; RA: Right atrium; LV: Left ventricle; RV: Right ventricle

Video 2: (A) Lung ultrasound showing decreased lung sliding (red arrow) on the left side. (B) Lung ultrasound showing normal lung sliding (green arrow) at the right side

\section{References}

1. Laursen $C B$, Sloth E, Lambrechtsen J, et al. Focused sonography of the heart, lungs, and deep veins identifies missed lifethreatening conditions in admitted patients with acute respiratory symptoms. Chest 2013;144(6):1868-1875. DOI: 10.1378/chest.130882.

2. Oks M, Cleven KL, Cardenas-Garcia J, et al. The effect of pointof-care ultrasonography on imaging studies in the medical ICU: a comparative study. Chest 2014;146(6):1574-1577. DOI: 10.1378/ chest.14-0728.

3. Volpicelli G, Elbarbary M, Blaivas M, et al. International liaison committee on lung ultrasound (ILC-LUS) for international consensus conference on lung ultrasound (ICC-LUS). international evidencebased recommendations for point-of-care lung ultrasound. Intensive Care Med 2012;38(4):577-591. DOI: 10.1007/s00134-012-2513-4.
4. Bouzat $P$, Francony $G$, Declety $P$, et al. Transcranial Doppler to screen on admission patients with mild to moderate traumatic brain injury. Neurosurgery 2011;68(6):1603-1609. DOI: 10.1227/ NEU.0b013e31820cd43e.

5. Troianos CA, Hartman GS, Glas KE, et al. Councils on intraoperative echocardiography and vascular ultrasound of the American Society of Echocardiography. Guidelines for performing ultrasound guided vascular cannulation: recommendations of the American Society of Echocardiography and the Society of Cardiovascular Anesthesiologists. J Am Soc Echocardiogr 2011;24(12):1291-1318. DOI: 10.1016/j.echo.2011.09.021.

6. COVID-19 Map- Johns Hopkins Coronavirus Resource Centre. (2020, October 28). Retrieved from https://coronavirus.jhu.edu/map.html.

7. Zieleskiewicz L, Muller L, Lakhal K, et al., CAR'Echo and AzuRea Collaborative Networks Point-of-care ultrasound in intensive care units: assessment of 1073 procedures in a multicentric, prospective, observational study. Intensive Care Med 2015;41(9):1638-1647. DOI: 10.1007/s00134-015-3952-5.

8. Gray R, Baldwin F, Bruemmer-Smith S. Diagnostic echocardiography in an unstable intensive care patient. Echo Res Pract 2015;2(1):K11-K16. DOI: 10.1530/ERP-14-0040.

9. Marcelino PA, Marum SM, Fernandes AP, et al. Routine transthoracic echocardiography in a general intensive care unit: an 18 month survey in 704 patients. Eur J Intern Med 2009;20(3):e37-e42. DOI: 10.1016/j.ejim.2008.09.015.

10. Who should not take Tocilizumab Syringe? (2020, October 28). Retrieved from https://www.webmd.com/drugs/2/drug-165324/ tocilizumab-subcutaneous/details/list-contraindications.

11. Singh $Y$, Tissot $C$, Fraga MV, et al. International evidence-based guidelines on point of care ultrasound (POCUS) for critically ill neonates and children issued by the POCUS working group of the European Society of Paediatric and Neonatal Intensive Care (ESPNIC). Crit Care 2020;24(1):65. DOI: 10.1186/s13054-020-2787-9.

12. Sjaus A, Fayad A. The use of subcostal echocardiographic views to guide the insertion of a right ventricular temporary transvenous pacemaker-description of the technique. J Cardiothorac Vasc Anesth 2019;33(10):2797-2803. DOI: 10.1053/j.jvca.2019.01.033.

13. Lichtenstein D, Goldstein I, Mourgeon E, et al. Comparative diagnostic performances of auscultation, chest radiography, and lung ultrasonography in acute respiratory distress syndrome. Anesthesiology 2004;100(1):9-15. DOI: 10.1097/00000542-20040100000006.

14. Bouhemad B, Brisson H, Le-Guen M, et al. Bedside ultrasound assessment of positive end-expiratory pressure-induced lung recruitment. Am J Respir Crit Care Med 2011;183(3):341-347. DOI: 10.1164/rccm.201003-03690C.

15. Bouhemad B, Liu ZH, Arbelot C, et al. Ultrasound assessment of antibiotic-induced pulmonary reaeration in ventilator-associated pneumonia. Crit Care Med 2010;38(1):84-92. DOI: 10.1097/ CCM.0b013e3181b08cdb.

16. Volpicelli G, Melniker LA, Cardinale L, et al. Lung ultrasound in diagnosing and monitoring pulmonary interstitial fluid. Radiol Med 2013;118(2):196-205. DOI: 10.1007/s11547-012-0852-4. 